\title{
Multi-site scheduling under production and transportation constraints
}

\author{
Fehmi H'Mida ${ }^{1}$, Pierre Lopez ${ }^{2,3}$ \\ ${ }^{1}$ UR MSSDT ESSTT, Université de Tunis - Tunis - Tunisia (fehmi.hmida@esti.rnu.tn) \\ ${ }^{2}$ CNRS, LAAS, 7 avenue du colonel Roche, F-31400 Toulouse, France (pierre.lopez@laas.fr) \\ ${ }^{3}$ Univ de Toulouse, LAAS, F-31400 Toulouse, France
}

\begin{abstract}
For the enterprises organized in several distributed production sites, usually, production scheduling models presume either an instantaneous delivery of products or an unlimited number of available vehicles for transporting products. However, the transportation of the intermediate products to the sites is an important activity within the whole process of manufacturing, and the efficient coordination of production and transportation becomes a challenging problem in the actual higher collaborative and competitive environments. This work focuses on the integrated production and transportation scheduling properly managing the resources capacity, material flows and temporal interdependencies between sites. A casestudy is reported and the industrial problem under consideration is modeled as a Constraint Satisfaction Problem (CSP). Besides scheduling under resource constraints, the model presented in this paper expands the packing problem to the area of transportation operations scheduling. It is implemented under the constraint programming language CHIP V5. The provided solutions determine values for the various variables associated to the production and transportation operations realized on the whole multi-site, as well as the curves with the profile of the total consumption of resources in time.
\end{abstract}

Keywords: Production and transportation scheduling, constraint programming, cumulative resource, packing problem. 


\section{INTRODUCTION}

A multi-site enterprise is a company with several distributed production sites, where sites are using the intermediate products of other sites to manufacture the products of the enterprise (Sauer and Appelrath, 2000). The scheduling problem is the temporal assignment of activities to resources where a number of goals and constraints have to be regarded.

For production scheduling, numerous researches have been proposed in the literature to address the problem in process industries. Various types of methods have been employed such as the mathematical programming approaches, heuristic and rule-based procedures. Extensive reviews of planning and scheduling methodologies, as well as contributions reported in the field, can be found in (Shah, 1998), (Pekny and Reklaitis, 1998), (Reklaitis, 2000), (Floudas and Lin, 2004) and (Méndez et al., 2006).

Usually, production scheduling models presume either an instantaneous delivery of products or an unlimited number of available vehicles for transporting products. However, the problem in which a limited number of vehicles available in a site can be found in most of the production sites, involving assignment, routing and timing decisions in a multi-site scheduling framework.

Thus, interacting production and transportation activities requires the consideration of additional features and constraints. Departure times of vehicles depend on the availability of products in site tied to the production scheduling defined, as well as on the availability of the assigned vehicle and on the capacities production of sites. The efficient coordination of production and transportation operations remains an open area for research (Erengüç et al., 1999; Sarmiento and Nagi, 1999; Chen, 2004; ElMaraghy and Mahmoudi, 2009). In general, literature related to the production-transport scheduling problems focuses mainly on the integration in strategic and tactical levels of analysis; the start times of transportation and production operations, the allocation of vehicles, the resource capacities in site and the products to be delivered, are issues rarely addressed jointly.

This work focuses on the integrated scheduling of the production and transportation operations, consuming resources (respectively machines and vehicles) with limited capacities. We propose a multi-site scheduling approach optimizing the end date of the production, while satisfying at the same time the constraints between operations and the resource constraints, 
that is, at every time, the sum of the resource consumptions for the operations should not exceed the production and the transportation capacities in sites. We model the problem in the CSP (Constraint Satisfaction Problem) form. The constraint programming paradigm consists in finding among the possible values of a set of variables those which satisfy all the constraints simultaneously. We are principally interested in the respect of the capacity resources and the synchronization operations, for jointly production and transportation scheduling in the multi-site. Besides scheduling under resource constraints, the model presented in this paper expands the packing problem to the area of transportation operations scheduling. Analogous to placing rectangular box onto a 3D space, we model the transportation multi-site scheduling by placing the transportation operations onto a space with three dimensions temporal, geographical and assignment. For solving, we implement the cumulative and packing global constraints concept, which provides a compact and concise description of the problem.

The remainder of the paper is organized as follows. The industrial problem considered is defined in Section 2. Section 3 recalls the basic principles of constraint programming and presents the cumulative and packing global constraints concept. Section 4 defines the parameters, variables and constraints of the industrial problem and formulates the multi-site scheduling model under production and transportation constraints. In Section 5, the proposed approach is implemented under the constraint programming language CHIP V5; some results are presented. Finally, Section 6 is devoted to the concluding remarks and some future research directions.

\section{INDUSTRIAL PROBLEM STATEMENT}

The industrial problem handled in this work appears as follows: 5 production sites appertaining to the same company, and realizing 11 types of products (Table 1). The manufacturing of a product requires a succession of production operations, realized each on a given site. The transfer between sites is provided by transportation operations (Figure 1). The term "operation" is employed to characterize a "workload". 


\begin{tabular}{|c|c|c|c|c|c|c|}
\hline Product & Quantity $\boldsymbol{Q}_{\boldsymbol{x}}$ & Site 1 & Site 2 & Site 3 & Site 4 & Site 5 \\
\hline A & 180 & $\mathrm{X}$ & & $\mathrm{X}$ & $\mathrm{X}$ & $\mathrm{X}$ \\
\hline $\mathrm{B}$ & 120 & $\mathrm{X}$ & & & & $\mathrm{X}$ \\
\hline $\mathrm{C}$ & 150 & $\mathrm{X}$ & & & & \\
\hline $\mathrm{D}$ & 130 & $\mathrm{X}$ & & $\mathrm{X}$ & $\mathrm{X}$ & $\mathrm{X}$ \\
\hline $\mathrm{E}$ & 140 & & $\mathrm{X}$ & $\mathrm{X}$ & $\mathrm{X}$ & $\mathrm{X}$ \\
\hline $\mathrm{F}$ & 150 & & $\mathrm{X}$ & & & $\mathrm{X}$ \\
\hline $\mathrm{G}$ & 20 & $\mathrm{X}$ & & & & \\
\hline M & 70 & & $\mathrm{X}$ & $\mathrm{X}$ & $\mathrm{X}$ & $\mathrm{X}$ \\
\hline N & 180 & $\mathrm{X}$ & & $\mathrm{X}$ & $\mathrm{X}$ & \\
\hline $\mathrm{O}$ & 100 & & $\mathrm{X}$ & $\mathrm{X}$ & $\mathrm{X}$ & $\mathrm{X}$ \\
\hline K & 30 & & $\mathrm{X}$ & $\mathrm{X}$ & $\mathrm{X}$ & $\mathrm{X}$ \\
\hline
\end{tabular}

Table 1: Quantity and process production of products

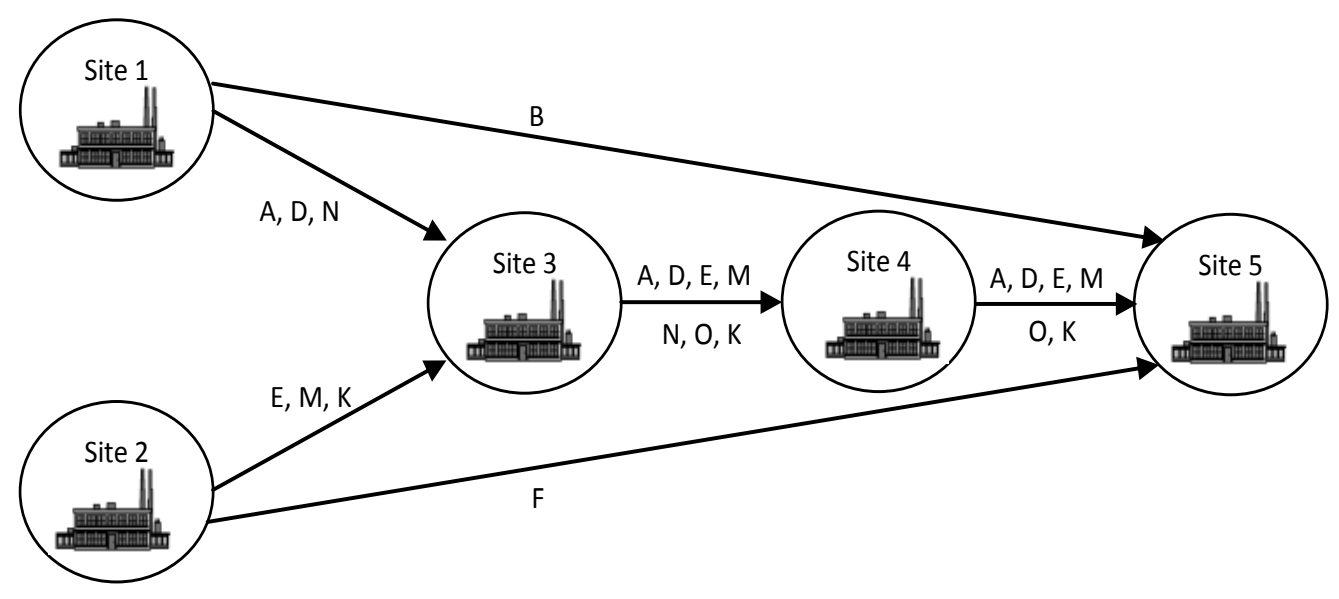

Figure 1: The multi-site company

A site is defined by its production capacity and its transportation capacity. The number of resources (i.e. of machines) in a site limits the production capacity (Table 2).

\begin{tabular}{|c|c|c|}
\hline Site & $\begin{array}{c}\text { Maximal number of } \\
\text { machines }\end{array}$ & $\begin{array}{c}\text { Maximal number of } \\
\text { trailers }\end{array}$ \\
\hline$S T_{1}$ & 4 & 5 \\
\hline$S T_{2}$ & 5 & 4 \\
\hline$S T_{3}$ & 3 & 5 \\
\hline$S T_{4}$ & 3 & 5 \\
\hline$S T_{5}$ & 3 & - \\
\hline
\end{tabular}

Table 2: Production and transportation capacity of sites 
The transportation is realized by vehicles composed by two types of resources: tractors and trailers (Figure 2). Trailers, properties of the company, are defined by their incorporation bases (sites), their availability over the planning period, and their maximal number in every site (Table 2). Every trailer can contain a maximal number of 40 products. A subcontracting transport company, supplying tractors to deliver trailers loaded from a site to the other one, ensures the transportation operations. The subcontractor can guarantee a simultaneous availability of 10 tractors at most. A tractor trip consists in towing a loaded trailer from its incorporation site to the delivery site followed by its delivery to the departure site (Table 3). The deliveries are realized by product.
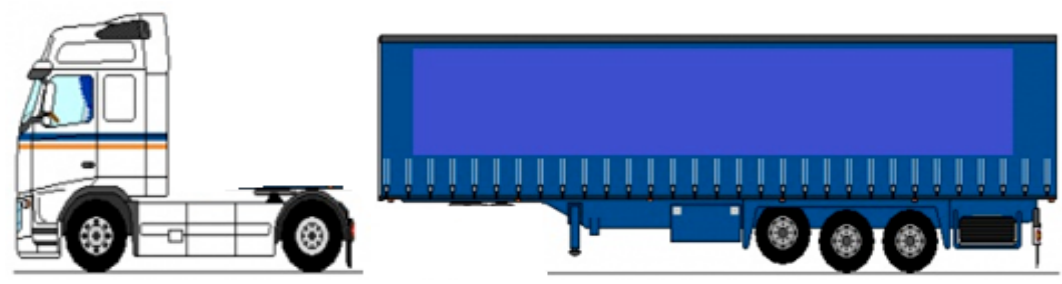

Figure 2: The transportation vehicle [Tractor + Trailer]

\begin{tabular}{|c|c|c|}
\hline $\begin{array}{c}\text { Origin } \\
\text { Site }\end{array}$ & $\begin{array}{c}\text { Destination } \\
\text { site }\end{array}$ & $\begin{array}{c}\text { Duration } \\
\text { (h) }\end{array}$ \\
\hline$S T_{1}$ & $S T_{3}$ & 8 \\
\hline$S T_{1}$ & $S T_{5}$ & 12 \\
\hline$S T_{2}$ & $S T_{3}$ & 5 \\
\hline$S T_{3}$ & $S T_{4}$ & 5 \\
\hline$S T_{2}$ & $S T_{5}$ & 10 \\
\hline$S T_{4}$ & $S T_{5}$ & 6 \\
\hline
\end{tabular}

Table 3: Transportation duration inter-site (round trip)

The elaboration of production plans of the 11 products for all the 5 sites supposes a horizontal synchronization of all the sites to assure the manufacturing and the provision of products in due course. This entails that the scheduling decisions integrate strictly the production constraints and the transportation constraints. In this treated problem, the decisions to make consist: 
-To determine for every site, the start dates of the production operations while respecting the limitation of the production resources capacity (number of machines in site)

-To determine for every site, the start dates of the transportation operations while respecting the limitation of the transportation resources capacity (number of trailers in site)

-To allocate tractors to the transportation operations within the limits of the simultaneous availability of 10 tractors, and by considering the geographical disparity of sites.

Thus, it is a matter of finding a solution that minimizes the production end date of all the products, by respecting at the same time precedence, cumulative and assignment constraints.

This multi-site scheduling problem is approached by considering the case of a company within which there is a central logistic department. It is a matter of distributing and coordinating production and transportation between sites while leaving to every site autonomy at the local scheduling level.

\section{CONSTRAINT PROGRAMMING}

Many decision problems in industry can be viewed as satisfiability or optimization problems. Different formulations (models) can be associated with the problem under study, and several criteria could be considered to evaluate a formulation. Two paradigms have reached a high degree of sophistication from the viewpoint of both theory and implementation: Constraint Programming (CP) and Mixed Integer Programming (MIP). The CP and MIP paradigms have strengths and weaknesses that can complement each other. The major difference between both techniques is the inference method. Inference is the act of revealing implicit constraints from the existing ones, in order to reduce the search space. On the one hand, $\mathrm{CP}$, through the use of sophisticated propagation techniques, privileges primal inference. On the other hand, MIP, through the techniques of relaxation and strengthening through cutting planes, privileges dual inference (Salvagnin, 2009).

In the last years, several researchers from the CP and MIP communities have investigated the possibility to integrate the methodologies proper to these paradigms (Hooker, 2006; Milano, 2003). Such an integration has the potential to yield important benefits, and the literature on this topic is recent but growing. 
In this work, we privileged the Constraint Programming approach, and particularly the use of the cumulative and packing global constraints, because it provides a relatively high level of abstraction and expressivity in the modelling phase. Moreover, $\mathrm{CP}$ offers a concise and integrated modelling of the presented scheduling problem and closer to natural language of the case study (straightforward and intuitive variables and constraints).

Constraint programming refers to the techniques dealing with constraint representation and exploitation. This paradigm potentially combines methods of operations research (e.g., graph theory, mathematical programming, combinatorial optimization methods) with tools resulting from artificial intelligence (e.g., filtering algorithms, instantiation heuristics, search schemes). Research carried out on constraint satisfaction problems (CSPs) has resulted in the development of effective models which are now widely used in various domains such as computer vision, robot or agent planning, scheduling, human resources management, supply chain, agronomy, diagnosis, or others (Gyssens et al., 1994; Van Hentenryck et al., 1992; Vargas, 1995; Chan and Chan, 2006).

\subsection{The CSP formalism}

A CSP is defined as a triplet $(X, D, C)$ (Montanari, 1974; Dechter, 2003) with:

- $X=(X 1, X 2, \ldots, X n)$ is the set of variables of the problem;

- $(D=D 1, D 2, \ldots, D n)$ is the set of domains. Each variable $X i \in X, 1 \leq i \leq n$, is associated with one domain $D i \in D$ which represents all the possible values for $X i$. These domains are finite, but of any kind, symbolic or numerical;

- $C=(C 1, C 2, \ldots, C k)$ is the set of constraints. Each constraint $C j$ is a relation between some variables of $X$. These constraints are of any kind, linear (e.g., $X 1+X 2 \leq 4)$ or nonlinear $(e . g ., X 3 \neq X 4)$.

Given a $\operatorname{CSP}(X, D, C)$, a feasible solution is an instantiation of all the variables, so that all the constraints are simultaneously satisfied.

\subsection{CSP solving}

General CSPs belong to the class of NP-complete problems. Their solving is based on the application of constraint propagation techniques (filtering phase) and on tree search (decisionmaking phase). 
Filtering phase: It consists in removing the values of the variables that have no chance to be among a solution. Within the filtering algorithms, the arc-consistency (AC) is probably the most used. This algorithm checks the consistency of a constraint between two variables of a CSP. Since the seminal AC-3 (Mackworth, 1977), many more powerful versions have been proposed (Mohr and Henderson, 1986; Bessière, 1994). However, the easy implementation of AC-3, its adaptability to broader frameworks than the classical CSP, as well as recent improvements of the basic version (Zhang and Yap, 2001; Bessière et al., 2005; Arangu et al., 2009) make this algorithm a widely used filtering technique.

Decision-making phase: It consists in finding a complete instantiation (a solution) satisfying all the constraints. Such an instantiation is carried out by means of various algorithms based on tree search, e.g., Backtrack (Bitner and Reingold, 1975), Limited Discrepancy Search (Harvey and Ginsberg, 1995), Randomization \& Restart (Gomes et al., 1998). Some of them are hybrid algorithms in the sense they perform a certain level of filtering on each variable instantiation in the tree expansion (Forward-Checking, Real-Full-Look-Ahead (Nadel, 1989), Maintaining Arc-Consistency (Sabin and Freuder, 1994)).

Last, let us mention that scanning the search space can be improved by ordering heuristics (order of the variable instantiations and choice of a value for a given variable).

\subsection{Global constraint}

Combinatorial problems generally present independent substructures easily identifiable, all of which being formulated by a group of constraints. This is the reason for introducing the concept of "global constraint". A global constraint is a subset of constraints, corresponding to a substructure of the original problem. Several types of global constraints have been developed: alldifferent, diffn, cycle, sequence, cumulative, etc. In the example of the global constraint "alldifferent $(X 1, \ldots, X n)$ ", each of the variables $X 1, \ldots, X n$ must take a different value, i.e., $X 1 \neq X 2 \neq \ldots \neq X n$. To each global constraint, at least one specific filtering algorithm is associated. A global constraint takes into account the group of constraints as a whole, in a more effective manner than standard propagation techniques applied to separate constraints. 


\subsubsection{Cumulative constraint}

Scheduling under resource constraints handles the problem taking, on the one hand, a set of tasks $i$ in which every task is defined by a start time $S_{i}$, a processing time $p_{i}$ as well as the quantity of resource consumption $r_{i, k}$, and, on the other hand, a limit of resource not exceeding $R_{k}$. This capacity limitation of resource $k$ imposes that at every time, the sum of the resource consumptions for the tasks in process should not exceed the resource capacity. It yields:

$\sum r_{i, k} \leq R_{k}$

$\sum_{i \in T k(t)}$

with $T_{k}(t)=\{i\}_{i=1 . . n}$ with $t \in\left[S_{i}, S_{i}+p_{i}\right)$.

This problem can be classified in the category of RCPSPs (Resource-Constrained Project Scheduling Problems), for which a variety of methods were employed for its resolution: linear programming, constraint programming, heuristics and metaheuristics, and tree search ((Baptiste and Le Pape, 1997; Beldiceanu et al., 1996; Dorndorf et al., 1999, Brucker et al., 1999; GOThA, 2006; Özdamar and Ulusoy, 1995) and more recently and very promisingly satisfiability-based solving (Coelha and Vanchoucke, 2011). In constraint programming, the cumulative global constraint (Aggoun and Beldiceanu, 1993) found in the RCPSP a particularly favourable field of application.

Form: Cumulative $\left(\left[S_{1}, \ldots, S_{n}\right],\left[p_{1}, \ldots, p_{n}\right],\left[E_{1, k}, \ldots, E_{n, k}\right],\left[r_{1, k}, \ldots, r_{n, k}\right], R_{k}, Z_{k}\right)$.

In the cumulative global constraint, a set of $n$ tasks has to be planned on resource $k ; E_{i, k}$ corresponds to the energy required and $Z_{k}$ the calculation of the completion time of the tasks on resource $k$ (Figure 3). It consists in the evaluation, for a given interval, of the sum of the resource consumptions required to perform the tasks; if this number exceeds the resource capacity then a contradiction is detected. 


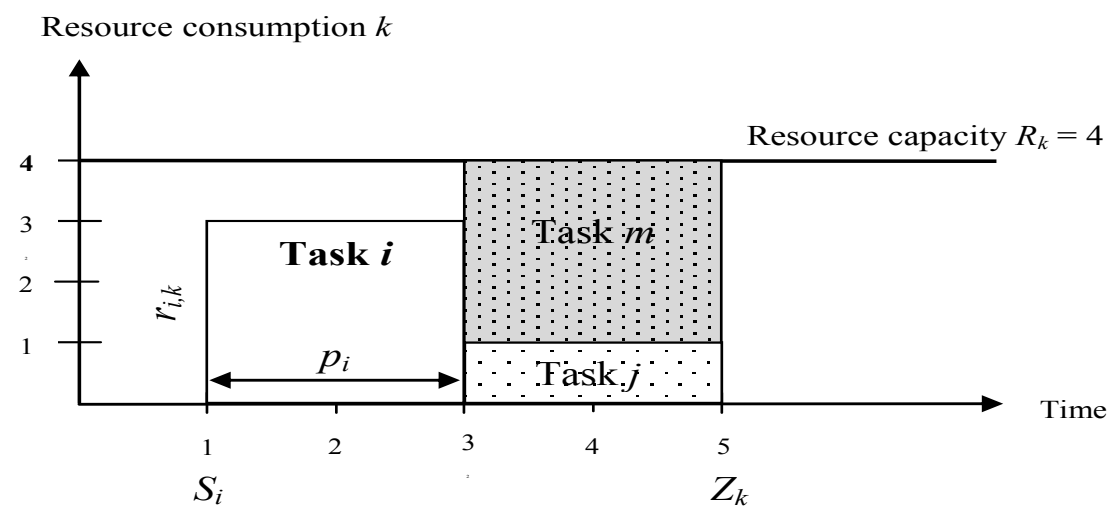

Figure 3: Example of a cumulative resource $k$

The first filtering algorithms were related to the notion of compulsory part of a task (Lahrichi, 1982). They compute a cumulated resource profile of all the compulsory parts of the tasks and prune the origins of the tasks with respect to this profile in order to not exceed the resource capacity. These methods are sometimes called timetabling. Even if these methods are quite local, i.e., a task has a non-empty compulsory part only when the difference between its latest start and its earliest start is strictly less than its duration, it scales well and is therefore widely used. Later on, more global algorithms based on the resource consumption of the tasks on specific intervals were introduced: energetic reasoning (Erschler and Lopez 1990), taskinterval (Caseau and Laburthe, 1996), cores-times (Klein and Scholl, 1999), etc.

\subsubsection{Packing constraint}

Given a set of components and one container (an available space area), a packing problem consists in finding the position variables of components in the container in effort to minimize a set of objectives, while respecting certain constraints. Every packing problem presents at least non-overlapping constraints between components and, in most of the cases, appertaining constraints to the container. They define the packing constraint.

Consider a list of $m$ rectangular boxes of dimension $n$ of which the sides are parallel to $n$ axes of a limited space $\left[E n d_{1}, \ldots, E n d_{n}\right]$. Every box is defined by the Cartesian coordinates $O_{i k}$ of its origin and its lengths $L_{i k}(i \in[1, m], k \in[1, n])$. The non-overlapping and appertaining constraints are respectively expressed by the formulas (2 and 3 ):

$$
\begin{aligned}
& \forall i \in[1, m], \forall j \in[1, m], i \neq j, \exists k \in[1, n] \text { such as } O_{i k} \geq O_{j k}+L_{j k} \vee O_{j k} \geq O_{i k}+L_{i k} \\
& \forall k \in[1, \mathrm{n}], i \in[1, m] \quad \text { Maximum }\left(O_{i k}+L_{i k}\right) \leq \text { End }_{k}
\end{aligned}
$$


Generally, components (boxes) are physical objects. In the literature, certain authors also consider abstract objects, presenting a direct analogy with the problem at hand (Dyckhoff, 1990). State of the art on packing problems shows that all types of optimization techniques (mathematical programming, constraint programming, heuristics and metaheuristics, etc.) were used for their resolution (Cagan et al., 2002; Dowsland, 1991).

In constraint programming, the packing global constraint noted "diffn" (Beldiceanu and Contejean, 1994) imposes the non-overlapping of a set of multidimensional rectangular boxes (Equation 2) and their appertaining in a limited space (Equation 3). The global constraint "diffn" imposes simultaneously these two types of constraints and then offers a more concise and integrated modelling of the packing problem.

Form: $\operatorname{diffn}([[O 11, \ldots, O 1 n, L 11, \ldots, L 1 n], \ldots,[O m 1, \ldots, O m n, L m 1, \ldots, L m n]],[$ End1, ..,Endn] $)$

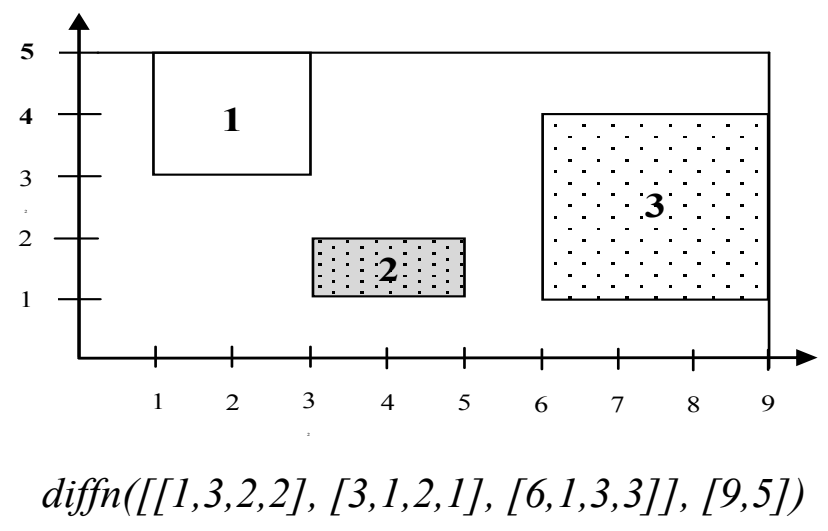

Figure 4: Illustration of the packing global constraint "diffn" (2 dimensions)

Checking whether a diffn constraint for which all variables are fixed is satisfied or not is related to the Klee's measure problem: given a collection of axis-aligned multi-dimensional boxes, how quickly can one compute the volume of their union. Then the diffn constraint holds if the volume of the union is equal to the sum of the volumes of the different boxes.

A first possible method for filtering is to use constructive disjunction. The idea is to try out each alternative of a disjunction (e.g., given two boxes $\mathrm{B}_{1}$ and $\mathrm{B}_{2}$ that should not overlap, we successively assume for each dimension that $\mathrm{B}_{1}$ finishes before $\mathrm{B}_{2}$, and that $\mathrm{B}_{2}$ finishes before $\mathrm{B}_{1}$ ) and to remove values that were pruned in all alternatives. For the two-dimensional case of diffn a second possible solution used in (Du Verdier, 1992) is to represent explicitly the twodimensional domain of the origin of each rectangle by a quadtree (Samet, 1989) and to accumulate all forbidden regions within this data structure. As for conventional domain 
variables, a failure occurs when a two-dimensional domain get empty. A third possible filtering algorithm based on sweep is described in (Beldiceanu and Carlsson, 2001).

\section{MULTI-SITE SCHEDULING MODEL}

In Section 2, we posed the industrial problem. In the sequel, we propose the associated multisite scheduling model in the form of a constraint satisfaction problem.

The proposed model is based around the definition of the parameters, the variables and the constraints translating the various characteristics related on times, the products and the logistical system. A solution consists in assigning a start time to each production and transportation operation by satisfying all the constraints.

\subsection{Parameters}

The data of the multi-site scheduling problem are represented by the following parameters:

- Elementary period of time $t$ : It is the unit of time in terms of scheduling,

- Decision horizon $H, t \in[1, H]$ : It is the period over which the scheduling decisions are made,

- The set of production sites $S T_{i}: S T=\left(S T_{1}, S T_{2}, S T_{3}, S T_{4}, S T_{5}\right)$,

- The set of products: $X=(A, B, C, D, E, F, G, M, N, O, K)$,

- The set of production operations $x^{i}: T=(A 1, A 3, A 4, A 5, B 1, B 5, C 1, D 1, D 3, D 4, D 5, E 2$, E3, E4, E5, F2, F5, G1, M2, M3, M4, M5, N1, N3, N4, O2, O3, O4, O5, K2, K3, K4, K5),

- The set of transportation operations $x^{i{ }^{\prime}}: T^{T}=(A 13, A 34, A 45, B 15, D 13, D 34, D 45, E 23$, E34, E45, F25, M23, M34, M45, N13, N34, O23, O34, O45, K23, K34, K45).

The achievement of each product requires the processing of a set of operations $x^{i}$ and $x^{i{ }^{\prime}}$. Each operation is performed on a different site. Example: Product $B$ requires the realization of 3 operations: $(B 1, B 15, B 5)$. B1 and B5, production operations, are respectively carried out on site 1 and site 5. B15 corresponds to the transportation operation from site 1 to site 5 . 
- Production and transport capacity, $R_{k}$ et $R^{T}{ }_{k}$ : They represent for each production site, respectively the maximal number of resources $k$ (machines), and the maximal number of resources $k^{T}$ (trailers) being able to be used during various periods $T$ (Table 2), $R_{1}=4 ; R_{2}=5 ; R_{3}=3 ; R_{4}=3 ; R_{5}=3 ; R_{1}^{T}=5 ; R_{2}^{T}=4 ; R_{3}^{T}=5 ; R_{4}^{T}=5$,

- Energy required $E_{x}{ }^{i}:$ It denotes the energy required to perform the production operation $x^{i}$ on unit resource $k$,

- Energy required $E_{x}^{i i}:$ It denotes the energy required to perform the transportation operation $x^{i i i^{\prime}}$ on unit resource $k^{T}$,

- Transport unit duration $D_{i i}$ : It corresponds to the time of one transportation operation $x^{i i^{\prime}}$ (round trip) between a site $S T_{i}$ and a site $S T_{i}$, (Table 3),

- Limit $\beta$ : It corresponds to the maximal number of tractors exploitable simultaneously. This value agreed suited with the subcontracting transport company corresponds to 10 .

\subsection{Variables}

The various variables that intervene in the scheduling model are the following:

- Start time $S x^{i}$ : variable corresponding to the start time of the production operation $x^{i}$,

- Start time $S x^{i i}$ : variable corresponding to the start time of the transportation operation $x^{i i}$

- Consumption $r_{x}^{i}$ : number of machines allocated to the production operation $x^{i}$,

- Consumption $r_{x}^{i i^{\prime}}$ : number of trailers allocated to the transportation operation $x^{i i}$,

- Duration $p x^{i}$ : duration of the production operation $x^{i}$,

- Duration $p x^{i i^{\prime}}:$ duration of the transportation operation $x^{i i^{\prime}}$,

- Completion time $Z_{i}$ : completion time of all production operations $x^{i}$ on site $S T_{i}$, $Z_{i}=\max \left(S x^{i}+p x^{i}\right)$ for $i \in\{1,2,3,4,5\}, x \in\{A, B, C, D, E, F, G, M, W, O, K\}$

Example: $Z_{1}=\max \left(S A^{l}+p A^{l}, S B^{l}+p B^{l}, S C^{l}+p C^{l}, S D^{l}+p D^{l}, S G^{l}+p G^{l}, S W^{l}+p W^{l}\right)$

- Completion time $Z_{i}^{T}$ : completion time of all transportation operations $x^{i i}$ from site $S T_{i}$ to sites $S T_{i}$,

$Z_{i}^{T}=\max \left(S x^{i i^{\prime}}+p x^{i i^{\prime}}\right)$ for $i \in\{1,2,3,4\}, i^{\prime} \in\{2,3,4,5\}$, 
and $x \in\{A, B, C, D, E, F, G, M, W, O, K\}$ with $i \neq i$ '

Example: $Z_{1}^{T}=\max \left(S A^{13}+p A^{13}, S B^{15}+p B^{15}, S C^{13}+p C^{13}, S N^{13}+p N^{13}\right)$

- Total completion time: completion time of all production and transportation operations on all sites,

$$
Z=\max \left(Z_{1}, Z_{2}, Z_{3}, Z_{4}, Z_{5}, Z_{1}{ }^{T}, Z_{2}{ }^{T}, Z_{3}{ }^{T}, Z_{4}{ }^{\mathrm{T}}\right)
$$

- $T x^{i i}$ : number of tractors transporting the trailers allocated to the transportation operation $x^{i i}$,

$T x^{i i \prime}=r_{x}{ }^{i i^{\prime}}$

- $L x^{i i^{\prime}}$ : index of the first tractor in the list of tractors allocated to the transportation operation $x^{i i}$,

$L x^{i i \prime} \in\{1,2, \ldots, 10\}$

- $W x^{i i}$ : number of round trips realized by tractors to assure the transportation operation $x^{i i}$,

$W x^{i i \prime} \in\{1,2, \ldots, 20\}$

\subsection{Constraints}

The problem is subject to five types of constraints: the energy constraints, the resource capacity constraints, the precedence constraints, the synchronization constraints, and the packing constraints.

\section{-Energy constraints:}

This corresponds to the energy consumption of an operation on a resource (Lopez et al., 1992). It expresses the existing relation between the variation of the operation duration $p x^{i}$ (respectively $\left.p x^{i i^{\prime}}\right)$ according to the number of resources $r_{x}^{i}\left(r e s p . r_{x}^{i{ }^{\prime}}\right)$ allocated for its processing.

For production operations $E_{x}^{i}=p x^{i} * r_{x}^{i}=$ constant

For transportation operations $E_{x}^{i i^{\prime}}=p x^{i i^{\prime}} * r_{x}^{i{ }^{\prime}}=$ constant

Figure 5 shows an example of a production operation $A^{1}$ with $E_{x}{ }^{i}=6$. In this case, the possible values of $p x^{i}$ and $r_{x}^{i}$, are: ((2.3), (3.2), (6.1)). The solution (2.3) means that production operation $A^{1}$ requires 3 resources during 2 time units. 


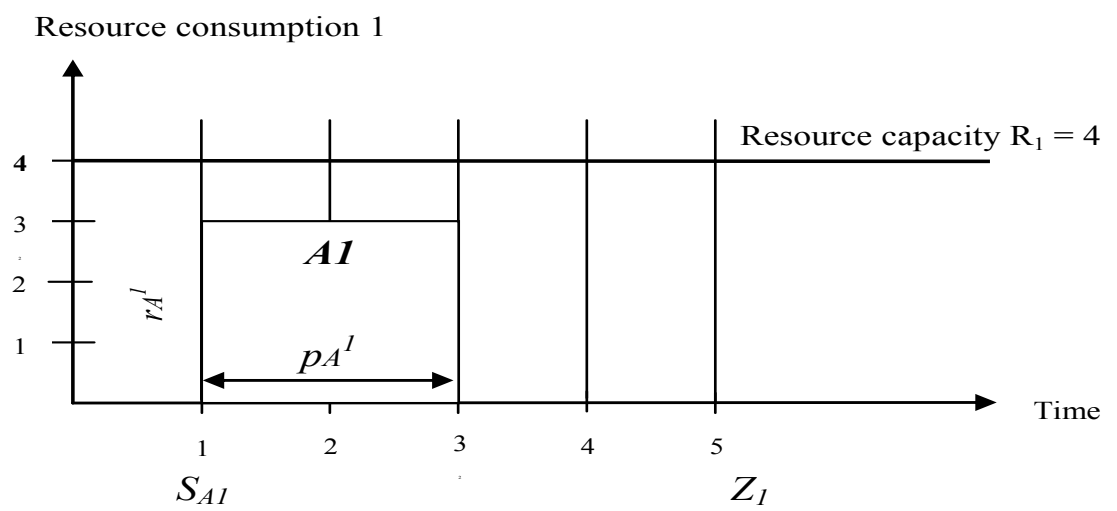

Figure 5: Example of a production operation $A^{1}\left(E_{A}{ }^{1}=6, p A^{1}=2, r_{A}{ }^{1}=3\right)$

- Capacity resource constraints:

For each resource $k$ (respectively $k^{T}$ ), they express the fact that, at every time, the total number of machines (resp. trailers) used by a set of operations $x^{i}$ (resp. $x^{i i^{\prime}}$ ) processed at time $t, S x^{i} \leq t \leq S x^{i}+p x^{i}$ (resp. $\left.S x^{i i^{\prime}} \leq t \leq S x^{i i^{\prime}}+p x^{i i^{\prime}}\right)$ does not exceed a certain capacity $R_{k}$ (resp. $R_{k}{ }^{T}$. They are used to model the cumulative resource constraints.

For production operations For $i \in\{1,2,3,4,5\}, k \in(1,2,3,4,5), x \in\{A, B, C, D, E, F$, $G, M, W, O, K\}, \forall t \in\left[\min \left(S x^{i}\right), \max \left(S x^{i}+p x^{i}\right)\right]$

$$
\sum_{S x^{i}<t<S x^{i}+p x^{i}} r_{x^{i}} \leq R_{k}
$$

For transportation operations For $i=\{1,2,3,4\}, i^{\prime}=\{2,3,4,5\}, k=(1,2,3,4), x \in\{A$, $B, C, D, E, F, G, M, W, O, K\}$ with $i \neq i, \forall t \in\left[\min \left(S x^{i i^{\prime}}\right), \max \left(S x^{i i^{\prime}}+p x^{i i^{\prime}}\right)\right]$

$$
\sum_{S x^{i i^{\prime}}<t<S x^{i i^{\prime}}+p x^{i i^{\prime}}} \leq R_{k}^{T}
$$

- Precedence constraints:

The precedence constraints between production operations are of two types; those corresponding to the process plan of a product and those imposed by the production manager. They have the form $i \rightarrow j$, prohibiting the start of the second operation, $j$, before the end of the first, $i$.

- Process plan constraints: for each product a process plan sets a sequence of production operations necessary for its realization. 


$$
S x^{j} \geq S x^{i}+p x^{i}
$$

For example, the process plan of product $A$ is: $(A 1, A 3, A 4, A 5)$. The associated precedence constraint states: $A 4 \rightarrow A 5$ is $S A^{5} \geq S A^{4}+p A^{4}$.

-Production constraints: some products must be processed before others (constraints imposed by the manager production). These inter-products constraints are expressed as follows:

$G \rightarrow E: S E^{3} \geq S G^{l}+p G^{l}$

- Synchronization constraints:

These constraints concern the synchronization of the production and transportation. Indeed, the transportation of every product $x$ from the departure site $S T_{i}$ to the following site $S T_{i}$, cannot begin before the end of production operation $x^{i}$ in the site $S T_{i}$. Also, the production of a product $x$ on a site $i^{\prime}$ can begin only after the delivery of all the quantity of Product $x$ coming from Site $i$, and given that every trailer can contain only a maximal number of products (40), it means that the delivery of the total quantity of a product can require several successive round trips. Constraint (16) constitutes a coordination rule in the definition of the master planning of the multi-site company.

$\forall i=\{1,2,3,4\}, i^{\prime}=\{2,3,4,5\}$ et $x \in\{A, B, C, D, E, F, G, M, W, O, K\}$ with $i \neq i$ '

$S x^{i i^{\prime}} \geq S x^{i}+p x^{i} \Lambda S x^{i^{\prime}} \geq S x^{i i^{\prime}}+p x^{i i^{\prime}}$

Furthermore, the duration of a transportation operation of a product $x$ must be multiplied by the associated transport unit duration. This means that it must be equal to the round trips $W x^{i i^{\prime}}$ multiplied by the transport unit duration $D_{i i}$ '.

$p x^{i i^{\prime}}=D_{i i^{\prime}}, W x^{i i^{\prime}}$

For example, the transportation duration of Product $B$ from the production site $S T_{1}$ towards $S T_{5}$ is equal to $W_{B}{ }^{15}$ (number of round trips) multiplied by 12 hours (transport unit duration): $p_{B}{ }^{15}=W_{B}{ }^{15} * 12$.

- Packing constraints: 
In a not conventional way, we exploit the packing constraints to model the scheduling of the transportation operations on the production sites. Concretely, the transportation operations correspond to rectangular boxes to position in a limited three-dimensional space (Figure 6):

- A geographical dimension allowing the localization of the departure site of a transportation operation,

- A temporal dimension allowing the localization of a transportation operation in the time,

- An assignment dimension indicating to which tractor(s) is assigned a transportation operation.

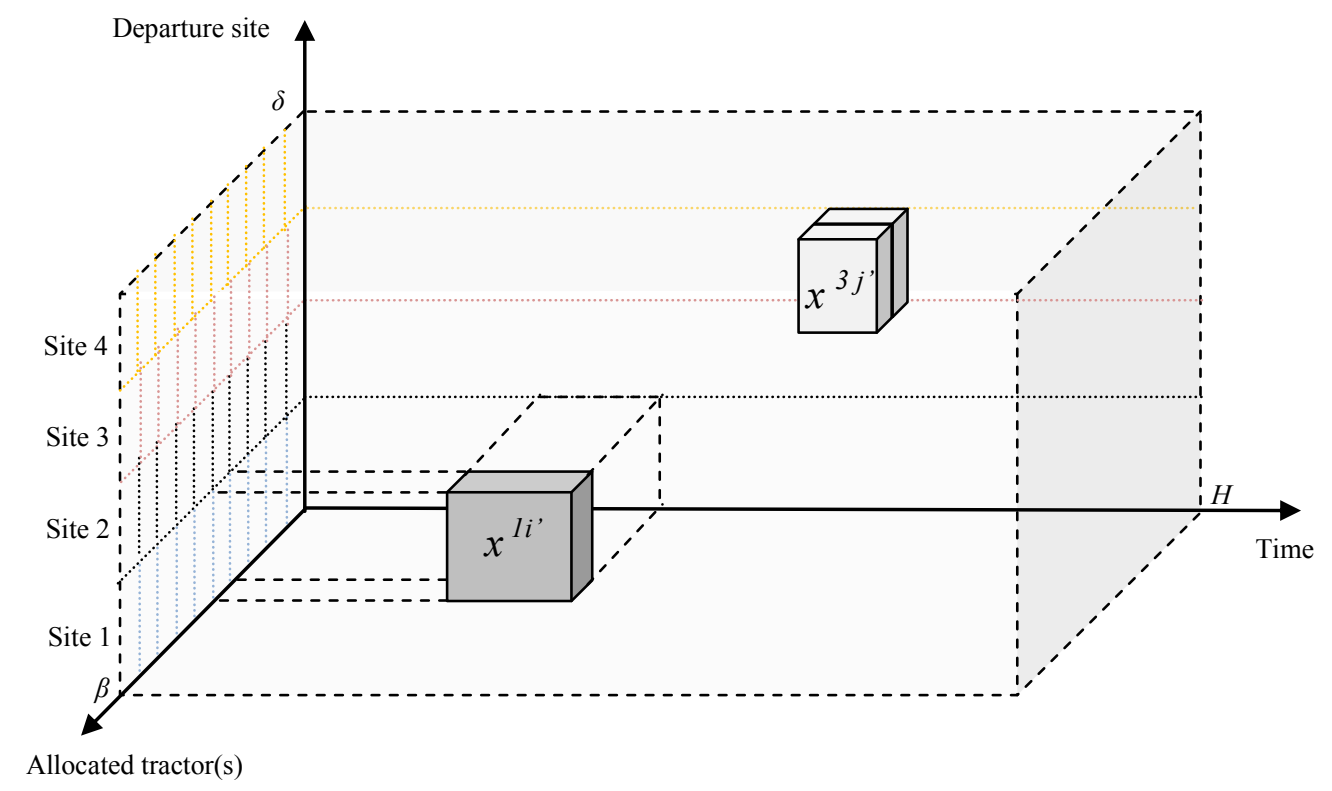

Figure 6: Positioning of transportation operations in the limited three dimensional space

In Figure 6, the transportation operation $x^{l i{ }^{\prime}}$ begins in a date $S x^{1 i{ }^{\prime}}$ from the site $S T_{1}$ to the site $S T_{i}$, with one tractor (and $r_{x}{ }^{1 i^{\prime}}=1, L x^{1 i^{\prime}}=5$ ). The transportation operation $x^{3 j^{\prime}}$ begins in a date $S x^{3 j^{\prime}}$ from the site $S T_{3}$ to the site $S T_{j^{\prime}}$ with 2 tractors (and $r_{x}^{3 j^{\prime}}=2, L x^{3 j^{\prime}}=1$ ). By combining the three dimensions, the objective is to place in the time every transportation operation, and on the other hand to assign to each a number of tractor(s), this without overlapping the operations, and within the limits of a space ( $\delta$ : departure sites; $\mathrm{H}$ : scheduling horizon and $\beta$ : maximal number of tractors).

The non-overlapping and appertaining constraints ( 2 and 3$)$ are translated in this model by the following equations:

- Non-overlapping constraints: 
$\forall x^{i i^{\prime}} \in T^{T}, \forall x^{i j j^{\prime}} \in T^{T}, x^{i i^{\prime}} \neq x^{i j^{\prime}}$ with $i$ and $j \in\{1,2,3,4\}$

$S x^{i j^{\prime}} \geq S x^{i i^{\prime}}+p x^{i i^{\prime}} \vee S x^{i i^{\prime}} \geq S x^{i j^{\prime}}+p x^{i j^{\prime}} \vee L x^{i j^{\prime}} \geq L x^{i i^{\prime}}+r_{x}^{i i^{\prime}} \vee L x^{i i^{\prime}} \geq L x^{i j^{\prime}}+r_{x} j^{\prime}$

- Appertaining constraints:

$\forall x^{i i} \in T^{T}$

$\operatorname{Maximum}\left(S x^{i i^{\prime}}+p x^{i i^{\prime}}\right) \leq H$

$\operatorname{Maximum}\left(L x^{i i^{\prime}}+r_{x}^{i i^{\prime}}\right) \leq \beta$

$\operatorname{Maximum}\left(Q^{i i^{\prime}}\right) \leq \delta$

Find a multi-site scheduling plan solution consists in determining the start time and the allocation of resources of each production and transportation operation, which satisfy simultaneously all the presented constraints: energy constraints, resource capacity constraints, precedence constraints, synchronization constraints, and packing constraints.

The objective function of the proposed model is to find an optimal multi-site scheduling plan that minimizes the Total completion time (completion time of all production and transportation operations on all sites),

$$
\operatorname{Min}\left[Z=\max \left(Z_{1}, Z_{2}, Z_{3}, Z_{4}, Z_{5}, Z_{1}^{T}, Z_{2}{ }^{T}, Z_{3}{ }^{T}, Z_{4}{ }^{\mathrm{T}}\right)\right]
$$

\section{EXPERIMENTS}

Various propagation and solving algorithms of CSPs have been integrated into Constraint Programming languages. Many environments have been developed and distributed. Among these environments, some were developed in a commercial perspective (e.g., CHIP, ILOGSolver), other are pure academic tools (e.g., Oz, Gnu-Prolog, CIAO SICStus). The scheduling model we developed was implemented with CHIP V5. The solving is based on the application of constraint propagation techniques (AC-3 arc-consistency filtering algorithm) and on tree search (Forward-Checking in the decision-making phase). For the ordering heuristics, we used the most_constrained and mindomain rules (most_constrained chooses the variable which has the smallest ratio of the number of values in its domain over the variable degree; mindomain fixes the variable with the smallest value in its domain). 


\subsection{Model implementation}

The implementation consists to declare the domain variables (decision), to post the constraints, and finally to enumerate and/or optimize the solutions. The variables are declared as domain variables that take their values in finite sets of integers. The constraints implemented are of two types. The constraints of the first type are written in form of arithmetic linear constraints. The second type uses the concept of global constraints. Therefore, the cumulative global constraint was used to integrate two specific constraints: the cumulative constraints and the energy consumptions, in turn, the packing global constraint was used to integrate two other particular constraints: the non-overlapping constraints and the appertaining constraints.

\subsubsection{Cumulative global constraints}

The cumulative constraint imposes that at each time $t$, if we consider all the present operations, the accumulation of their consumed resources quantity should not exceed the limit of available resources. It is expressed in CHIP V5 by the following predicate:

$$
\text { Cumulative (+Starts, +Durations, +Surfaces, +Resources, +High, +End) }
$$

Thus, we associated, in every production site, two cumulative constraints, the first relative to the production capacity and the second relative to the transportation capacity. We present, below, that relative to the limitation of the production capacity on site $S T_{l}$.

$$
\text { Cumulative }\left(S x^{1}, p x^{1}, E_{x}{ }^{l}, r_{x}{ }^{l}, R 1, Z 1\right)
$$

The variables $S x^{1}=[S A 1, S B 1, S C 1, S D 1, S G 1, S N 1], p x^{1}=[p A 1, p B 1, p C 1, p D 1, p G 1, p N 1]$, $r_{x}{ }^{1}=[r A 1, r B 1, r C 1, r D 1, r G 1, r N 1]$ and $Z 1$ correspond respectively to start times $S x^{l}$, durations $p x^{l}$, number of machines allocated $r_{x}{ }^{1}$ of the production operations $A 1, B 1, C 1$, $D 1, G 1, N 1$, and to the completion time $Z 1$ of these six operations on site $S T_{1}$. The constants $E_{x}{ }^{l}=[E A 1, E B 1, E C 1, E D 1, E G 1, E N 1]$ and $R 1$ correspond respectively to the energy required $E_{x}{ }^{1}$ for the realization of each operation $A 1, B 1, C 1, D 1, G 1, N 1$, and to the production capacity $R l$ in number of machines on site $S T_{1}$. The rest of the cumulative constraints relative to the production capacities of sites $S T_{2}, S T_{3}, S T_{4}$ and $S T_{5}$ take the same form.

We present below the cumulative constraint relative to the capacity of the transportation products from the production site $S T_{1}$. 


$$
\text { Cumulative }\left(S x^{1 i^{\prime}}, \mathrm{px}^{1 i^{\prime},}, E_{x}{ }^{1 i^{\prime}}, r_{x}{ }^{1 i^{\prime}}, R^{T} 1, Z^{T} 1\right)
$$

The variables $S x^{1 i^{\prime}}=[S A 13, S B 15, S D 13, S N 13], p x^{1 i^{\prime}}=[p A 13, p B 15, p D 13, p N 13]$, $r_{x}{ }^{l{ }^{\prime}}=[r A 13, r B 15, r D 13, r N 13]$ and $Z^{T} 1$ correspond respectively to start times $S x^{1 i^{\prime}}$, durations $p x^{1 i}$, number of trailers allocated $r_{x}{ }^{1{ }^{\prime}}$ of the transportation operations $A 13, B 15$, D13, N13 and the completion time $Z^{T} 1$ of these four operations from site $S T_{1}$. The constants $E_{x}{ }^{1 i^{\prime}}=[E A 13, E B 15, E D 13, E N 13]$ and $R^{T} 1$ correspond respectively to the energy required $E_{x}{ }^{l i}$ for the realization of each operation $A 13, B 15, D 13, N 13$ and the transportation capacity $R^{T} 1$ in number of trailers on the site $\mathrm{ST}_{1}$. The rest of the cumulative constraints relative to the transportation capacities of sites $S T_{2}, S T_{3}$ and $S T_{4}$ take the same form.

According to its commercial description, the processing of cumulative global constraints in CHIP V5 is performed by about twenty of methods. Among the known and effective methods, we can particularly note the energy reasoning and the timetable constraints. The first allows, on the basis of balance between the consumptions of a resource by activities over a time interval and the energy offered by this resource on the same interval, to determine a lower bound on the amount of the resource that can be used (Lopez et al., 1992). The second has the same goal, but it gets on the basis of constraints called timetable, based on the concept of compulsory part (Lahrichi, 1982), interval of time over which the task is necessarily processed.

\subsubsection{Packing global constraints}

The packing constraint imposes that, at each time $t$, the transportation operations (rectangular boxes) do not overlap, and do not exceed the limited three-dimensional space. It is expressed in CHIP V5 by the following predicate:

$$
\text { diffn(+Rectangles, }+ \text { End })
$$

Therefore we associated to all the transportation operations the following packing global constraint:

$$
\operatorname{diffn}\left(\left[\left[S x^{i i^{\prime}}, Q^{i i^{\prime}}, L x^{i i^{\prime}}, p x^{i i^{\prime}}, 1, r_{x}^{i i^{\prime}}\right]\right],[H, \delta, \beta]\right)
$$

In the three-dimensional space (temporal, geographical and tractors allocation), the position elements of every transportation operation $x{ }^{i i^{\prime}}$ are its origins and its lengths. The origins are: the variable $S x^{i i^{\prime}}$ (start operation), the parameter $Q^{i i^{\prime}}$ (departure site, $Q^{i{ }^{\prime}}=i$ ) and the variable $L x^{i i}$ ' (tractor index), and the lengths of the transportation operation are: 
the variable $p x^{i i}$ ' (operation duration), the value 1 (a single site of departure) and the variable $r_{x}^{i i}$ (number of tractors allocated to the operation). The space domain is limited by the scheduling horizon $H$, the number of sites $\delta$ and the number of tractors exploitable simultaneously $\beta$. The filtering algorithm applied for the diffn constraint is based on sweep, and it is described in (Beldiceanu \& Carlsson, 2001).

\subsection{Results}

The visualization of the consumption on each resource is information making it possible to undergo the satisfaction of all constraints of the problem, and particularly the packing and cumulative global constraints. The presentation of the results wants to be didactic; we then present as an example only the cumulative production and transportation curve of site $S T_{1}$, and the three-dimensional Gantt chart of the total transportation operations. The curve and analysis presented in this section relate to the optimal solution.

\subsubsection{Production operations}

The production capacity of site $S T_{1}$ is equal to 4 . It realizes the 6 production operations: $A 1$, $B 1, C 1, D 1, G 1$, and $N 1$. The following table presents the solution values of the operations $x^{1}$ realized on site $S T_{1}$.

\begin{tabular}{|c|c|c|c|c|}
\hline $\begin{array}{c}\text { Operation } \\
\boldsymbol{x}^{\boldsymbol{1}}\end{array}$ & $\begin{array}{c}\text { Energy } \\
\text { required } \\
\boldsymbol{E}_{\boldsymbol{x}}{ }^{1}\end{array}$ & $\begin{array}{c}\text { Start } \\
\text { times } \\
\boldsymbol{S} \boldsymbol{x}^{\boldsymbol{1}}\end{array}$ & $\begin{array}{c}\text { Operation } \\
\text { duration } \\
\boldsymbol{p} \boldsymbol{x}^{\boldsymbol{1}}\end{array}$ & $\begin{array}{c}\text { Machine } \\
\text { number } \\
\boldsymbol{r}_{\boldsymbol{x}}{ }^{\boldsymbol{1}}\end{array}$ \\
\hline $\mathrm{A} 1$ & 24 & 6 & 8 & 3 \\
\hline $\mathrm{B} 1$ & 19 & 14 & 19 & 1 \\
\hline $\mathrm{C} 1$ & 24 & 14 & 24 & 1 \\
\hline $\mathrm{D} 1$ & 29 & 6 & 29 & 1 \\
\hline $\mathrm{G} 1$ & 15 & 1 & 5 & 3 \\
\hline $\mathrm{N} 1$ & 30 & 14 & 30 & 1 \\
\hline
\end{tabular}

Table 4: Values of the variables production operations realized on site $S T_{1}$

The production cumulative curve of site $S T_{1}$ is presented in Figure 7. The X-axis presents the time (in hours) and the $\mathrm{Y}$-axis the number of machines. The profile visualizes the evolution of resource consumption over time. The red horizontal line indicates the maximum capacity of site $S T_{1}\left(R_{1}=4\right)$. The curve is composed by stacked rectangles associated to operations $A 1, B 1$, $C 1, D 1, G 1$, and N1. Each rectangle is characterized by one duration $p x^{1}$ and a number of 
consumed resource $r_{x}{ }^{l}$. The combination of these rectangles gives the profile of the total machine consumption over time.

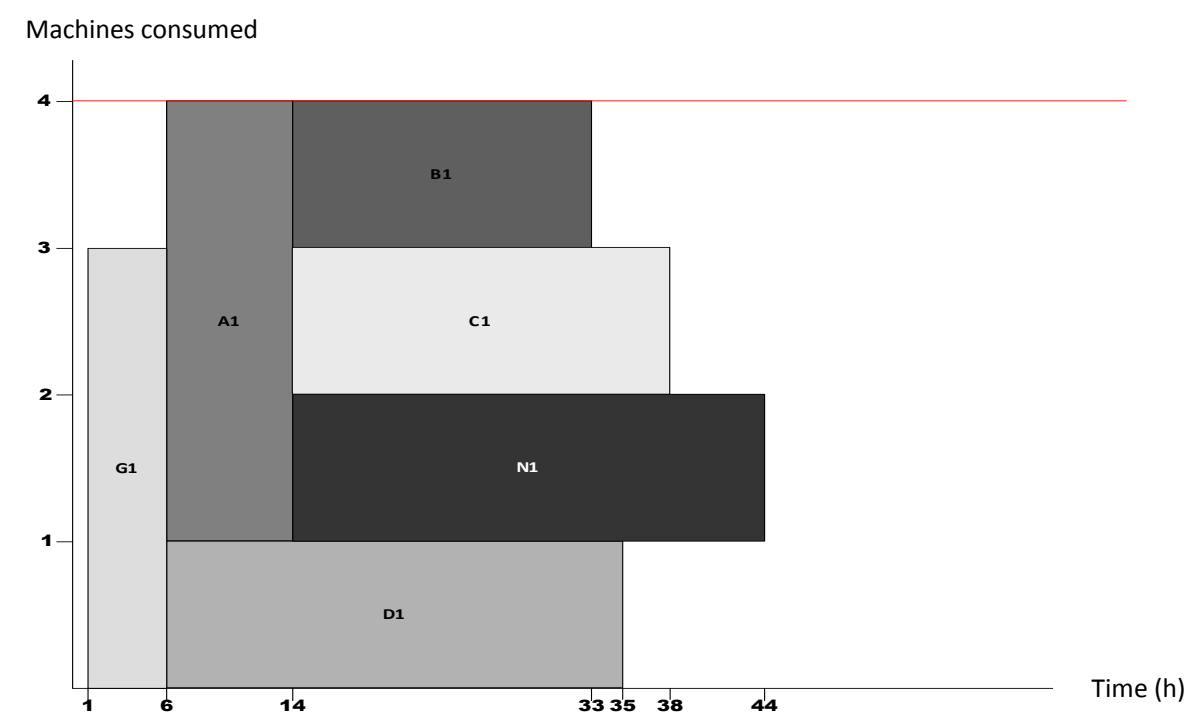

Figure 7: Cumulative production curve on site $S T_{l}\left(R_{l}=4\right)$

\subsubsection{Transportation operations}

The transportation capacity of site $S T_{1}$ is equal to 5 trailers. They are used for the 4 transportation operations: $A 13, B 15, D 13$ and N13. The following table presents the solutions values of the operations $x^{1 i}$.

\begin{tabular}{|c|c|c|c|c|}
\hline $\begin{array}{c}\text { Operation } \\
x^{1 i}\end{array}$ & $\begin{array}{c}\text { Energy required } \\
E_{x}{ }^{l i}\end{array}$ & $\begin{array}{l}\text { Start times } \\
\qquad S x^{I i}\end{array}$ & $\begin{array}{c}\text { Operation duration } \\
\qquad x^{l i}\end{array}$ & $\begin{array}{c}\text { Trailer number } \\
r_{x}^{l i}{ }^{\prime}\end{array}$ \\
\hline A13 & 40 & 14 & 8 & 5 \\
\hline B15 & 36 & 33 & 36 & 1 \\
\hline D13 & 32 & 35 & 16 & 2 \\
\hline N13 & 40 & 69 & 8 & 5 \\
\hline
\end{tabular}

Table 5: Values of the variables transportation operations from site $S T_{1}$

The transportation cumulative curve of site $S T_{1}$ is presented in Figure 8. The X-axis presents the time (in hours) and the Y-axis the number of trailers used by each transportation operation. The red horizontal line indicates the maximum number of trailers in site $S T_{1}$ $\left(R_{1}^{T}=5\right)$. 


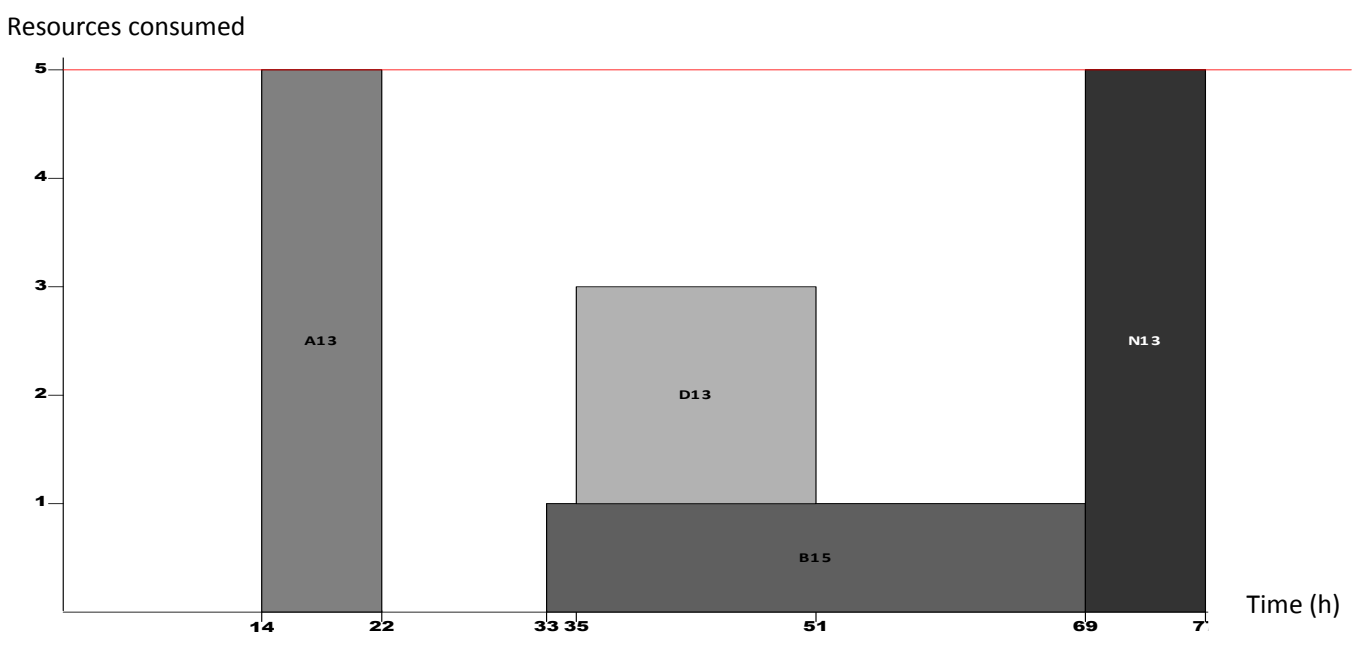

Figure 8: Cumulative transport curve of site $S T_{1}\left(R_{1}^{T}=5\right)$

\subsubsection{Three-dimensional packing}

The transportation operations are assured by sets (trailer + tractor). The cumulative constraint allows in particular calculating schedules according to the limitation and availability of the resources (machines and trailers). On the other hand, it does not determine how to specify the assignment of trailers to shared tractors. Hence, the implementation of the packing constraint allows us to provide this complementarity and to assure the position without overlapping of the transportation operations in the threedimensional space (time, sites, and tractors).

The 3D space is limited by the values $H=100, \delta=5$ and $\beta=10$, corresponding to the scheduling horizon, number of sites and number of tractors, respectively. Table 6 presents the values solutions of the transportation operations $x^{i i \prime}$ for the 11 products between the 5 production sites.

\begin{tabular}{|c|c|c|c|c|c|c|}
\hline \multirow{2}{*}{$\begin{array}{c}\text { Transportation } \\
\text { operation } \\
x^{i i^{\prime}}\end{array}$} & \multicolumn{3}{|c|}{ Operation origins $x^{i i^{\prime}}$} & \multicolumn{3}{|c|}{ Operation dimensions $x^{i i^{\prime}}$} \\
\cline { 2 - 7 } & $\begin{array}{c}\text { Start } \\
S^{p, i i^{\prime}}\end{array}$ & $\begin{array}{c}\text { Site } \\
Q^{i i^{\prime}}\end{array}$ & $\begin{array}{c}\text { Tractor } \\
\text { index } \\
L x^{i i^{\prime}}\end{array}$ & $\begin{array}{c}\text { Duration } \\
p x^{i i^{\prime}}\end{array}$ & $\begin{array}{c}\text { Number of } \\
\text { sites } \\
(\text { const }=1)\end{array}$ & $\begin{array}{c}\text { Number of } \\
\text { tractors } \\
r_{x}^{i i^{\prime}}\end{array}$ \\
\hline $\mathrm{A} 13$ & 14 & 1 & 1 & 8 & 1 & 5 \\
\hline $\mathrm{B} 15$ & 33 & 1 & 6 & 36 & 1 & 1 \\
\hline $\mathrm{D} 13$ & 35 & 1 & 7 & 16 & 1 & 2 \\
\hline $\mathrm{N} 13$ & 69 & 1 & 1 & 8 & 1 & 5 \\
\hline $\mathrm{E} 23$ & 45 & 2 & 1 & 5 & 1 & 4 \\
\hline
\end{tabular}




\begin{tabular}{|c|c|c|c|c|c|c|}
\hline F25 & 52 & 2 & 7 & 10 & 1 & 4 \\
\hline M23 & 15 & 2 & 6 & 10 & 1 & 1 \\
\hline O23 & 6 & 2 & 7 & 15 & 1 & 1 \\
\hline K23 & 6 & 2 & 1 & 5 & 1 & 1 \\
\hline A34 & 32 & 3 & 1 & 5 & 1 & 5 \\
\hline D34 & 56 & 3 & 1 & 10 & 1 & 2 \\
\hline E34 & 56 & 3 & 3 & 10 & 1 & 2 \\
\hline M34 & 37 & 3 & 5 & 10 & 1 & 1 \\
\hline N34 & 82 & 3 & 1 & 5 & 1 & 5 \\
\hline O34 & 37 & 3 & 9 & 15 & 1 & 1 \\
\hline K34 & 21 & 3 & 7 & 5 & 1 & 1 \\
\hline A45 & 50 & 4 & 1 & 6 & 1 & 5 \\
\hline D45 & 78 & 4 & 6 & 12 & 1 & 2 \\
\hline E45 & 72 & 4 & 6 & 6 & 1 & 4 \\
\hline M45 & 57 & 4 & 5 & 12 & 1 & 1 \\
\hline O45 & 62 & 4 & 10 & 18 & 1 & 1 \\
\hline K45 & 37 & 4 & 1 & 6 & 1 & 1 \\
\hline
\end{tabular}

Table 6: Values of the variables transportation operations between the five production sites

We present in Figure 9 a geometrical representation of the solution. The coordinates of the transportation operations on the 3 axes (Table 6) reflect their positions.

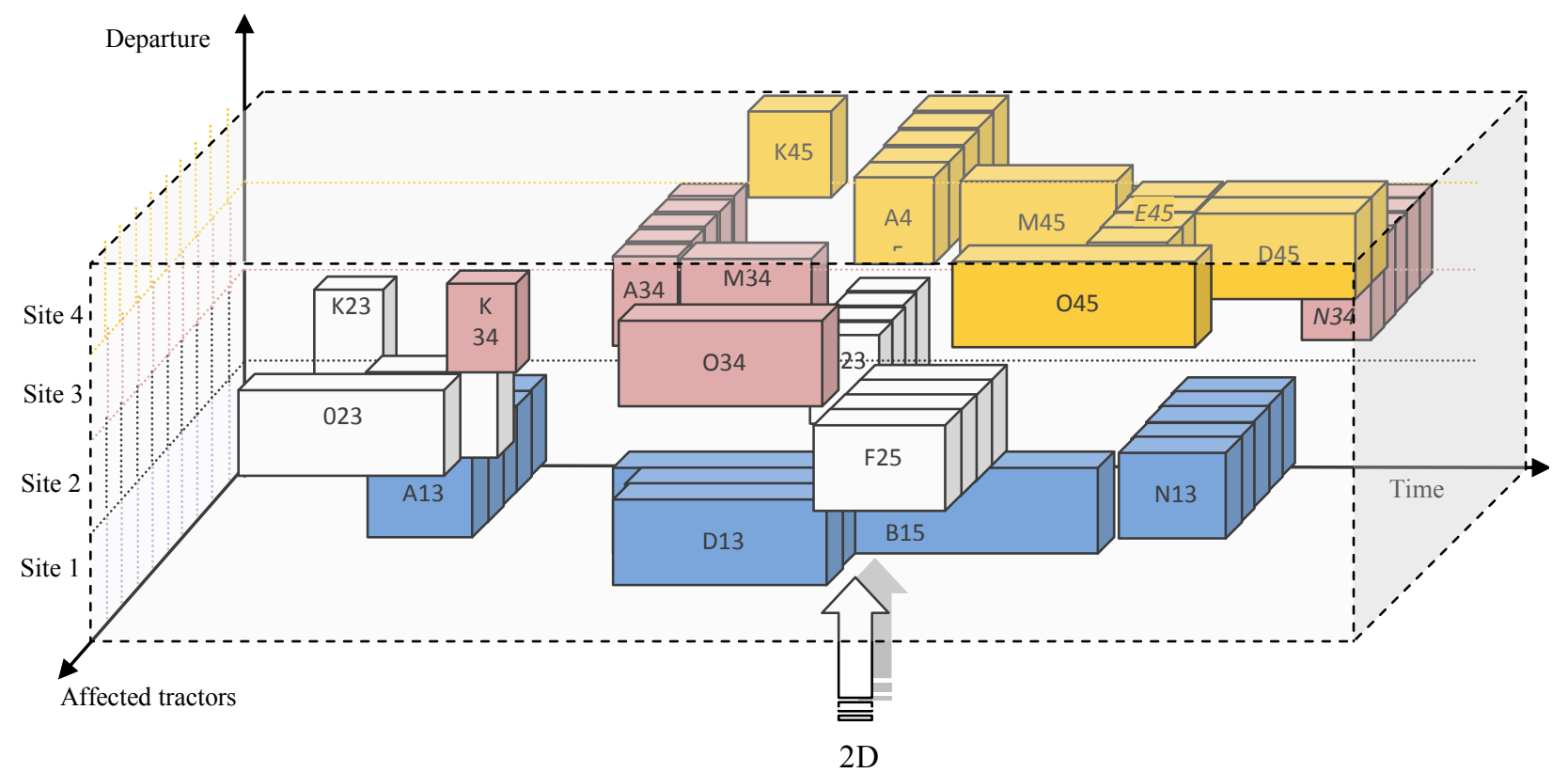

Figure 9: 3D view of the transportation operations between the sites production 


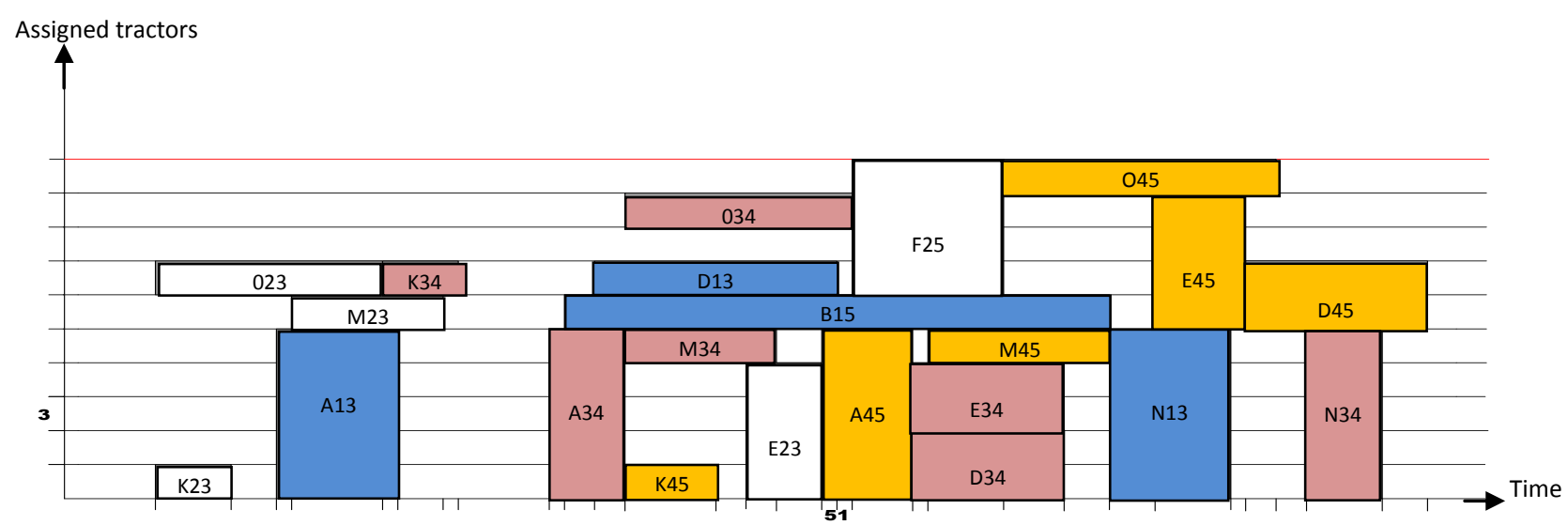

Figure 10: 2D view of the transportation operations between the sites production

These two figures (9 and 10) show, for every operation, which tractor(s) is allocated, from which date and site, and on how long. As an example, Operation F25 is assured by 4 tractors simultaneously, from the hour 52 and the site 2. Its duration is 10 hours. The respect for the capacity limitation of trailers on every site, the sharing of the 10 tractors and the non-overlapping of the transportation operations are satisfied.

\section{CONCLUSIONS}

The proposed multi-site scheduling model is based on a constraint satisfaction approach. It formalizes a set of decision variables to be managed and a set of constraints to be satisfied. In this framework, we have developed an integrated production/transportation operations model and implemented the associated cumulative and packing global constraints. The model takes into account the existing alternatives to the duration of an operation according to the number of resources used for its realization, while respecting the capacity of production and transportation resources in each site. The solving provides the optimal solution that determines the minimum time required to complete producing and delivering all the considered products over all sites. It integrates the production/transportation operations and schedules them jointly in a coordinated way. The different elements presented in the model were implemented through the constraint programming system CHIP V5. The results are delivered in numerical form and in the form of curves tracing, for a given site, the profile of 
the total consumption of resources over time. 2D and 3D view of transportation operations scheduling over all sites are also presented.

Further works plan to take account of the dynamic features of scheduling. That leads us to adopt an approach which is based on the Dynamic Constraint Satisfaction Problem formalism. The objective is to avoid starting again research from scratch after each change in the problem definition. Moreover, the more or less precise knowledge of the decision variables implies their controllability. This leads us to consider Conditional Constraint Satisfaction Problem with variables and constraints dependent on a situation and having conditions of presence.

\section{REFERENCES}

Aggoun, A., \& Beldiceanu, N., (1993). Extending CHIP in order to solve complex scheduling and placement problems. Mathematical and Computer Modelling, 17(7), 57-73.

Arangu, M., Salido, M.A., \& Barber, F., (2009). AC3-OP: An Arc-Consistency Algorithm for Arithmetic Constraints, Proceedings of the 12th International Conference of the Catalan Association for Artificial Intelligence (pp. 344-348), Vilar Rural de Cardona (El Bages), Cardona, Spain.

Baptiste, P., Le Pape, C., \& Nuijten, W., (2001). Constraint-Based Scheduling: Applying Constraint Programming in Scheduling Problems, Kluwer Academic Publishers.

Beldiceanu, N. \& Contejean, E., (1994). Introducing Global Constraints in CHIP. Mathematical Computer Modelling, 20(12), 97-123.

Beldiceanu, N., \& Carlsson, M., (2001). Sweep as a Generic Pruning Technique Applied to the Non-Overlapping Rectangles Constraints. 7th International Conference on Principles and Practice of Constraint Programming, Springer, 377-391. 
Beldiceanu, N., Bourreau, E., Rivreau, D., \& Simonis, H., (1996). Solving resourceconstrained project scheduling with CHIP. Proceedings of the 5th International Workshop on Project Management and Scheduling (pp. 35-38), EURO PMS, Poznan, Poland.

Bessière, C., (1994). Arc-consistency and arc-consistency again. Artificial Intelligence, 65(1), 179-190.

Bessière, C., Régin, J.-C., Yap R., \& Zhang, Y., (2005). An optimal coarse-grained arc consistency algorithm. Artificial Intelligence, 165(2), 165-185.

Bitner, J. \& Reingold, E., (1975). Backtracking programming techniques. Communications of the ACM, 18(11), 651-656.

Brucker, P., Drexl, A., Möhring, R., Neumann, K., \& Pesch, E., (1999). Resource-constrained project scheduling problem: Notation, classification, models and methods. European Journal of Operational Research, 112(1), 3-41.

Cagan, J., Shimada, K. \& Yin S., (2002). A survey of computational approaches to threedimensional layout problems. Computer-Aided Design, 34(8), 597-611.

Caseau, Y., \& Laburthe, F., (1996). Cumulative Scheduling with Task Intervals. Joint International Conference and Symposium on Logic Programming (JICSLP'96), MIT Press.

Chan, F. T. S., \& Chan, H., K., (2006). A simulation study with quantity flexibility in a supply chain subjected to uncertainties. International Journal of Computer Integrated Manufacturing, 19(2), 148-160.

Chen, Z.L., (2004). Integrated production and distribution operations: Taxonomy, models and review, Kluwer Academic Publishers (Chapter 17).

Coelhoa, J., \& Vanhoucke. M., (2011). Multi-mode resource-constrained project scheduling using RCPSP and SAT solvers, European Journal of Operational Research, 213(1), 73-82. 
Dechter, R., (2003). Constraint Processing. Morgan Kaufmann, San Francisco.

Dorndorf, U., Huy Phan, T., \& Pesch, E., (1999). A Survey of Interval Capacity Consistency Tests for Time- and Resource-Constrained Scheduling. In Project Scheduling - Recent Models, Algorithms and Applications, J. Weglarz (Ed). Kluwer Academic Publishers, 213238.

Dowsland, W.B., (1991). Three dimensional packing - solutions approaches and heuristic development. International Journal of Production Research, 29(8), 1673-1685.

Du Verdier, R., (1992). Résolution de problèmes d'aménagement spatial fondée sur la satisfaction de contraintes. Validation sur l'implantation d'équipements électroniques hyperfréquences. Ph.D. Thesis, Lyon I University, France.

Dyckhoff, H., (1990). A typology of cutting and packing problems. European Journal of Operational Research, 44(2), 145-159.

ElMaraghy, H. A., \& Mahmoudi, N., (2009). Concurrent design of product modules structure and global supply chain configurations. International Journal of Computer Integrated Manufacturing, 22(6), 483-493.

Erschler, J., \& Lopez, P., (1990). Energy-based approach for task scheduling under time and resource-constraints. 2nd International Workshop on Project Management and Scheduling (pp. 115-121), Compiègne, France.

Erengüç, S.S., Simpson, N.C. \& Vakharia, A.J., (1999). Integrated production/distribution planning in supply chains: An invited review. European Journal of Operational Research, $115,219-236$. 
Floudas, C.A. \& Lin, X., (2004). Continuous-time versus discrete-time approaches for scheduling of chemical processes: A review. Computers and Chemical Engineering, 28(11), 2109-2129.

Gomes, C., Selman, B., \& Kautz, H., (1998). Boosting Combinatorial Search Through Randomization. Proceedings of the $15^{\text {th }}$ National Conference on Artificial Intelligence (pp. 431-437), Madison, WI, USA.

GOThA, Working group on "Gestion de projet sous contraintes de ressources". Problème de gestion de projet à contraintes de ressources : approches et méthodes de résolution. http://www.ocea.li.univ-tours.fr/eocea/rcpsp, 2006 (in French).

Gyssens, M., Jeavons, P.G., \& Cohen, D.A., (1994). Decomposing constraint satisfaction problems using database techniques. Artificial intelligence, 66(1), 57-89.

Harvey, W. \& Ginsberg, M., (1995). Limited Discrepancy Search. Proceedings of the 14th International Joint Conference on Artificial Intelligence (pp. 607-613), Montreal, Canada.

Hooker J. N., (2006). Integrated Methods for Optimization. Springer.

Lahrichi, A., (1982). Scheduling: the notions of hump, compulsory parts and their use in cumulative problems. C. R. Acad. Sci., Paris, 294, 209-211.

Lock, H. C. R., (1996). An Implementation of the cumulative Constraint. Technical report, University of Karlsruhe.

Lopez, P., Erschler, J., \& Esquirol, P., (1992). Ordonnancement de tâches sous contraintes : une approche énergétique. Automatique, Productique, Informatique Industrielle, 26, 453-481.

Mackworth, A. K., (1977). Consistency in networks of relations. Artificial Intelligence, 8(1), 99-118. 
Méndez, C.A., Cerdá, J., Grossmann, I.E., Harjunkoski, I. \& Fahl, M., (2006). State-of-the-art review of optimization methods for short-term scheduling of batch processes, Computers and Chemical Engineering, 30 (6/7), 913-946.

Milano M., (2003). Constraint and Integer Programming: Toward a Unified Methodology. Kluwer Academic Publishers.

Mohr, R. \& Henderson, T., (1986). Arc and path consistency revisited. Artificial Intelligence, 28(2), 225-233.

Montanari, U., (1974). Network of constraints: Fundamental properties and applications to picture processing. Information Science, 7, 95-132.

Nadel, B., (1989). Constraint Satisfaction Algorithms. Computational Intelligence, 5, 188299.

Özdamar, L. \& Ulusoy, G., (1995). A survey on the resource constrained project scheduling problem. IIE Transactions, 27, 574-586.

Pekny, J. F. \& Reklaitis, G.V., (1998). Towards the convergence of theory and practice: A technology guide for scheduling/planning methodology. Proceedings of the $3^{\text {rd }}$ Conference Foundations of computer-aided process operations, AIChE symposium, 320(94), 91-111.

Reklaitis G.V., (2000). Overview of planning and scheduling technologies. Latin American Applied Research, 30, 285-293.

Sabin, D. \& Freuder, E., (1994). Contradicting conventional wisdom in constraint satisfaction. Proceedings of the $11^{\text {th }}$ European Conference on Artificial Intelligence (pp. 125-129), Amsterdam, The Netherlands.

Salvagnin D., (2009). Constraint programming techniques for mixed integer linear programs. 
$\mathrm{PhD}$ thesis. University of Padova, Italy.

Samet, H., (1989). The design and analysis of spatial data structures. Addison-Wesley.

Sarmiento, A.M., \& Nagi, R., (1999). A review of integrated analysis of production Distribution systems. IIE Transactions, 31, 1061-1074.

Sauer, J. \& Appelrath, H. J., (2000). Integrating Transportation in a Multi-Site Scheduling Environment, Proceedings of the $33^{\text {rd }}$ Hawaii International Conference on System Sciences (HICSS-33), IEEE Computer Society.

Shah, N., (1998). Single and multisite planning and scheduling: Current status and future challenges. Proceedings of the $3^{\text {rd }}$ Conference Foundations of Computer-Aided Process Operations Foundations of computer-aided process operations, AIChE symposium, 320(94), 75-90.

Van Hentenryck, P., Simonis, H., \& Dincbas, M., (1992). Constraint satisfaction using constraint logic programming. Artificial intelligence, 58, 113-159.

Vargas, C., (1995). Modélisation du processus de conception en ingénierie des systèmes mécaniques : Mise en oeuvre basée sur la propagation de contraintes, $\mathrm{PhD}$ thesis. Ecole Normale Supérieure de Cachan.

Zhang, Y. \& Yap, R., (2001). Making AC-3 an Optimal Algorithm. Proceedings of the 17th International Joint Conference on Artificial Intelligence, Seattle, WA, USA. 